\title{
Correlação entre Propriedades Reológicas e Ópticas de Filmes Tubulares de Polietileno Linear de Baixa Densidade com Diferentes Distribuições de Ramificações Curtas
}

\author{
Glayton M. Basso, Pedro I. Paulin-Filho, Rosario E. S. Bretas \\ Departamento de Engenharia de Materiais, UFSCar
}

Alessandro Bernardi Braskem, Triunfo, $R S$

\begin{abstract}
Resumo: Devido a suas excelentes propriedades mecânicas o polietileno linear de baixa densidade, PELBD, tem ganhado cada vez mais importância na indústria de embalagens. Essas propriedades são resultado de sua estrutura molecular, da existência de ramificações curtas, SCB, e da distribuição dessas ramificações, DSCB. Junto com o peso molecular, PM, e sua distribuição, DPM, as ramificações curtas também afetam o processo de sopro de filmes influenciando na solidificação do filme até a linha de neve, já que a cinética de cristalização é dependente da distribuição destas ramificações. Assim, este trabalho teve como objetivo verificar a influência da DSCB do PELBD nas propriedades reológicas e óticas de filmes tubulares. Para isso, três PELBD foram escolhidos; dois sintetizados com catalisador Ziegler-Natta e um com catalisador metaloceno, de peso molecular ponderal médio, $\mathrm{M}_{\mathrm{w}}$, similar e DSCB diferentes.

Observou-se que a DSCB exerce influência nas propriedades reológicas que refletem a elasticidade do material como a primeira diferença de tensões normais $\mathrm{N}_{1}$, o módulo de armazenamento $\mathrm{G}^{\prime}(\omega)$, e a deformação recuperável $\gamma_{r}$; entretanto na viscosidade em função da taxa de cisalhamento $\eta(\dot{\gamma})$, e no módulo de perda $G$ "' $(\omega)$, a diferença nessa distribuição não foi percebida. Observou-se também que quanto maior a deformação recuperável dos PELBD, menores foram as estruturas cristalinas formadas o que diminuiu a opacidade dos filmes. Porém essa proporcionalidade manteve-se até uma determinada $\gamma_{r}$ mínima, acima da qual a fratura do fundido se sobrepôs à recuperação da deformação e a opacidade voltou a aumentar.
\end{abstract}

Palavras-chave: Polietileno linear de baixa densidade, filmes soprados, deformação recuperável, opacidade.

\section{Correlation Between Rheological and Optical Properties of LLDPE Blown Films with Different Distributions of Short Chain Branching}

Abstract: Linear low-density polyethylene, LLDPE, has increased its market share in the packaging industry due to its excellent mechanical properties. These properties are the result of its molecular structure due to its short chain branching, $\mathrm{SCB}$, and their distribution, DSCB. Together with the molecular weight and the distribution of molecular weights, the SCB affects the blown film process because it interferes in the film solidification and crystallization kinetics up to the freezing line. The objective of this work was to verify the influence of the DSCB of LLDPE in the rheological and optical properties of blown films. Three LLDPE, synthesized with Ziegler-Natta and metallocene catalysts, were studied, all with similar weight average molecular weight, $\mathrm{M}_{\mathrm{w}}$, but with different DSCB. It was observed that the DSCB influenced the rheological properties that reflect the material elasticity, such as the first normal tension difference, $\mathrm{N}_{1}(\dot{\gamma})$, the storage modulus $\mathrm{G}^{\prime}(\mathrm{w})$ and the recoverable strain $\gamma_{\text {; }}$, however, the rheological properties that reflect the viscosity, such as the shear viscosity $\eta(\dot{\gamma})$ and the loss modulus G'(w), were not affected. In addition, the higher the $\gamma_{\mathrm{r}}$ of the LLDPE, the lower the crystalline structures of the films, which decreased their opacity. However this proportionality was kept up to a given value of $\gamma_{\mathrm{r}}$ above which the melt fracture dominated the recoverable strain and the opacity increased again.

Keywords: LLDPE, blown films, recoverable strain, opacity.

\section{Introdução}

Devido a suas excelentes propriedades mecânicas o PELBD tem ganhado cada vez mais importância na indústria de embalagens. Essas propriedades são o resultado de sua estrutura molecular, da existência de ramificações curtas, SCB e da distribuição dessas ramificações, DSCB. Junto com o PM e sua distribuição, DPM, as ramificações curtas também afetam o processo de sopro de filmes devido a sua influência na cinética de cristalização. 
Dependendo do tipo de catalisador, tecnologia do reator e condições de processo, resinas de PELBD de densidades similares podem apresentar quantidades e distribuições de ramificações curtas (inter ou intramoleculares) diferentes. Essas diferenças em ordem molecular provocam diferenças em propriedades como resistência ao impacto, resistência à fratura, à solda quente, extraíveis de hexano ${ }^{[1]}$ e cinética de cristalização ${ }^{[2]}$.

A maior viscosidade e maior susceptibilidade à fratura do fundido fazem com que o processamento do PELBD seja mais difícil em comparação com o polietileno de baixa densidade, PEBD. O PELBD apresenta resistência à tração e dureza menores e maior resistência ao impacto e rasgamento de filmes conforme aumenta o teor de ramificações ${ }^{[3]}$.

A produção de filmes tubulares por sopro de qualquer polímero envolve submetê-lo a vários tipos de deformações sob diferentes gradientes de temperatura. Dentro da matriz espiral de sopro da extrusora, como mostrado na Figura $1^{[4]} \mathrm{O}$ polímero fundido flui sobre taxas de deformação predominantemente de cisalhamento. Logo, propriedades reológicas em cisalhamento como a viscosidade $\eta(\dot{\gamma})$ e a elasticidade do fundido, descrita pela primeira diferença de tensões normais $\mathrm{N}_{1}(\dot{\gamma})=\left(\tau_{11}-\tau_{22}\right)$ são as controladoras do fluxo dentro da matriz.

A baixas taxas de cisalhamento $\dot{\gamma}$, a superfície do extrudado apresenta-se lisa. Porém, se $\dot{\gamma}$ for muito elevada pode ocorrer

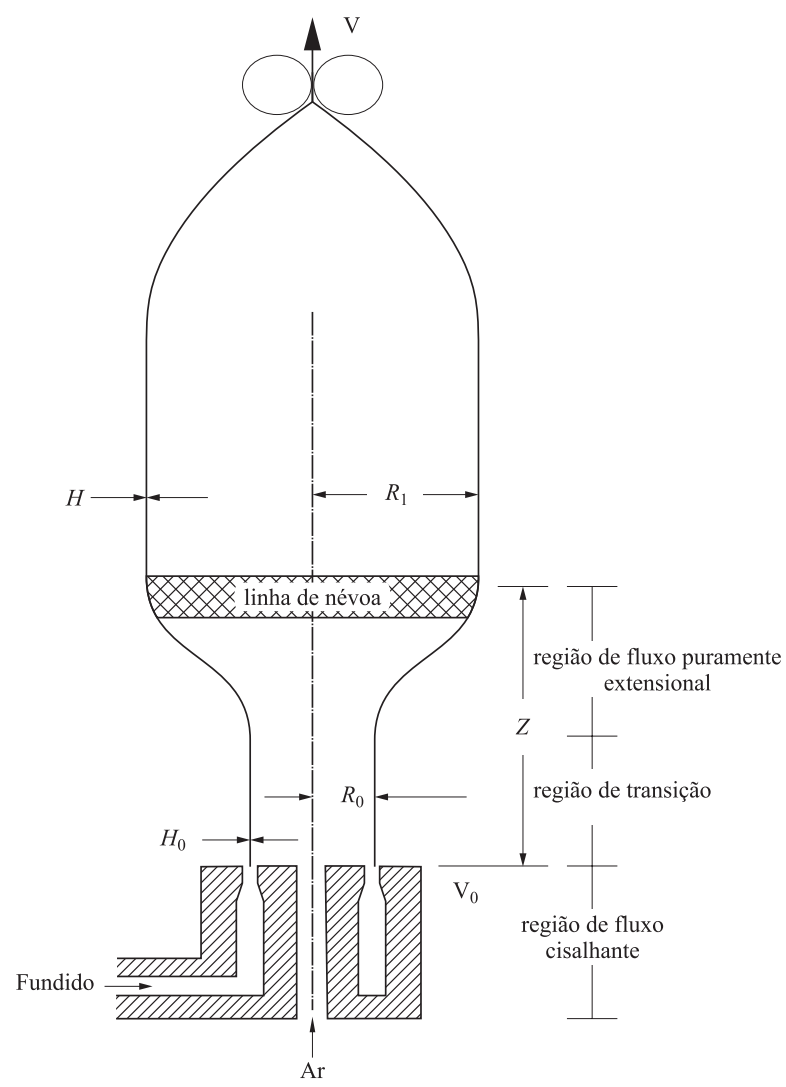

Figura 1. Esquema detalhado de uma extrusão de filmes tubulares ${ }^{[4]}$. $\mathrm{Z}=$ altura da linha de névoa; $\mathrm{V}=$ velocidade do filme após saída dos rolos; $\mathrm{V}=$ velocidade do filme na saída da matriz; $\mathrm{R}_{\mathrm{f}}=$ raio final do filme soprado; $\mathrm{R}_{\mathrm{o}}=$ raio do filme na saída da matriz; $\mathrm{H}=$ espessura final do filme; $\mathrm{H}_{\mathrm{o}}=$ espessura do filme na saída da matriz. a fratura do fundido, na qual irregularidades grosseiras ao longo da seção transversal do extrudado se desenvolvem. Sabe-se que a qualidade ótica dos filmes se deteriorará quando as taxas de produção (ou cisalhamento) aumentam. Assim, torna-se necessário conhecer a taxa de cisalhamento crítica, $\dot{\gamma}$, na qual o polímero começa a fraturar.

Na saída da matriz observa-se a ocorrência do inchamento do extrudado (a espessura da parede do filme é maior do que a espessura dos "lábios" da matriz). Esse inchamento acarreta também alterações nas propriedades óticas, e desta forma deve ser quantificado.

No interior da matriz o fundido estará fluindo devido a um gradiente de pressão. Quando o fundido deixa a matriz, a pressão ou tensão cai para zero. Assim, a cristalização do polímero ocorrerá sob a influencia de tensões biaxiais provenientes do ar interno e do estiramento pelos rolos, sob um elevado gradiente de temperatura. Logo, a morfologia e conseqüentemente as propriedades óticas do filme serão dependentes dos tempos de relaxação do fundido após a saída da matriz. Quanto mais rapidamente o fundido relaxar e mais rapidamente recuperar a deformação, mais rapidamente ele começará a cristalizar sem a influencia destas tensões biaxiais. Desta forma, propriedades reológicas em regime transiente de cisalhamento, como a deformação recuperável após a retirada de uma dada tensão, $\gamma_{r}(\tau, t)$ controlaram esta morfologia ${ }^{[5]}$.

Em relação ao processo em si, sabe-se ainda que a estabilidade da bolha durante o sopro pode ser correlacionada ao módulo de armazenamento G' do polímero fundido; quanto maior G' (maior elasticidade) maior será a estabilidade da bolha ${ }^{[6]}$.

Após a linha de névoa, a solicitação elongacional comandará a deformação no polímero. Assim, a propriedade reológica mais importante nesta etapa é a viscosidade em função da taxa de elongação $\bar{\eta}_{\mathrm{E}}$.

A influência dos parâmetros de processamento nas propriedades óticas e mecânicas de filmes tubulares de PELBD tem sido bastante estudada ${ }^{[7]}$. Tem se verificado que quanto maior a temperatura do fundido na saída da matriz, $T_{m}$, maior é a resistência ao impacto por dardo, maior o módulo secante na direção da máquina, DM e transversal a esta, DT, e maior a resistência ao rasgo na DM. Porém o aumento em $\mathrm{T}_{\mathrm{m}}$ provoca um aumento na opacidade total, porque aumenta o grau de cristalinidade e a opacidade interna.

Sukhadia et al. ${ }^{[8,9]}$ fizeram estudos correlacionando $\gamma_{\mathrm{r}}$ com a opacidade de filmes de PELBD, este último polimerizado com diferentes catalisadores (metaloceno, Ziegler Natta e Cr). Observou-se que a $\gamma_{\mathrm{r}}$ e a DPM aumentavam na seguinte ordem: m-PELBD $<$ ZN-PELBD $<$ Cr-PELBD. Também foi observado que quanto menor o valor de $\gamma_{\mathrm{r}}$ maior a opacidade; porém, esta proporcionalidade se mantinha até um determinado valor a partir do qual a opacidade voltava a aumentar novamente com o aumento de $\gamma_{\mathrm{r}}$. A elevada opacidade a baixos $\gamma_{\mathrm{r}}$ foi atribuída ao rápido relaxamento durante a formação da estrutura. Conforme $\gamma_{\mathrm{r}}$ aumentava, maior era a tendência à formação de estruturas lamelares orientadas e menor era a opacidade. Entretanto, após um certo valor de $\gamma_{\mathrm{r}}$, o tempo de relaxação ficava 
muito elevado, o que possibilitava o aparecimento de fenômenos reológicos como o inchamento do extrudado e a fratura do fundido que provocavam então novamente um aumento na opacidade do filme resultante. Os autores observaram ainda que quanto maior a incorporação de ramificações longas, LCB, menor era a $\gamma_{\mathrm{r}}$ e o tempo de relaxação.

Guerrini ${ }^{[6]}$ realizou estudo variando a porcentagem de PELBD em blendas com PEBD e também observou que quanto menor a $\gamma_{\mathrm{r}}$, maior a tendência à formação de estruturas esferulíticas, o que resultava em maior opacidade, contrastando com a estrutura lamelar orientada, preferencialmente formada quando o material apresentava altos valores de $\gamma_{\mathrm{r}}$. Kazatchkov et al..$^{[10]}$ observaram ainda uma correlação entre a taxa e a tensão crítica de cisalhamento para o aparecimento de fratura do fundido no PELBD em função do PM e DPM. Observaram que a taxa crítica $\dot{\gamma}_{c}$ diminuía com o aumento do tempo de relaxação (aumento do PM).

O PELBD apresenta dependência do tamanho da fratura do fundido (amplitude da deformação) com $\dot{\gamma}$ o que faz com que seus defeitos de extrusão fiquem perceptíveis a taxas mais baixas ${ }^{[11,12]}$.Tem sido verificado que o aumento da temperatura provoca redução do tamanho da fratura. Santamaría et al. ${ }^{[13]}$ estudaram o retardamento do aparecimento da pele de cação para amostras de m- PELBD diminuindo a temperatura da matriz. Verificaram a influencia do PM e quantidade de ramificações curtas na temperatura de extrusão de amostras com superfície lisa $\left(\mathrm{T}_{\mathrm{s}}\right)$. Em amostras de diferentes PM e quantidade de ramificações curtas similares não foi verificado efeito significativo nesta $T_{s}$. Entretanto, observou-se que quando a quantidade de ramificações curtas aumentava e o PM permanecia constante, a $\mathrm{T}_{\mathrm{s}}$ diminuía. Logo a DSCB pode influenciar as propriedades reológicas do PELBD.

Neste trabalho, a influencia da DSCB nas propriedades reológicas mais importantes durante o sopro de filmes tubulares de PELBD foi estudada e correlacionada com as propriedades óticas dos mesmos.

\section{Experimental}

\section{Materiais}

A Tabela 1 apresenta os PELBD utilizados; os PELBDZN-1 e ZN-2 foram sintetizados pela Braskem, enquanto o m-PELBD pela Exxon. A densidade foi obtida usando uma coluna de gradiente de densidade Davenport NENE,com isopropanol e água, a $23{ }^{\circ} \mathrm{C}$. O índice de fluidez, IF, foi medido num MFI Meltflow, usando 2,16 kg, a $190{ }^{\circ} \mathrm{C}$. Os pesos moleculares médios ponderais, $\mathrm{M}_{\mathrm{w}}$, numérico, $\mathrm{M}_{\mathrm{n}}$, e z-médio, $\mathrm{M}_{\mathrm{z}}$, foram medidos num cromatógrafo de permeação a gel Waters Modelo 150C, com coluna padrão de estireno, $1,2,4$ triclorobenzeno como solvente, a $140{ }^{\circ} \mathrm{C}$.

\section{Medida de Distribuição de ramificações curtas, DSCB}

A DSCB destes polímeros foi medida utilizando a técnica Crystaf da Polymer Char. A DSCB refere-se à distribuição da concentração de ramificações curtas nas moléculas de polietileno. As técnicas de medida da DSCB separam as moléculas de acordo com a quantidade de ramificações curtas que elas possuem, não se importando com a massa molar de cada molécula, ao contrário das técnicas de medida de DPM que separam as moléculas de acordo com as massas molares das mesmas não se importando com a quantidade de ramificações curtas destas moléculas.

No PELBD a incorporação de co-monômero nas cadeias do PE linear resulta em irregularidades (cadeias laterais) que modificam a habilidade de cristalização do polímero. Negligenciando a pequena influência do $\mathrm{PM}^{[14]}$, a cristalização em solução do PELBD irá segregar os cristais de acordo com a quantidade de co-monômero ou ramificação.

A técnica Crystaf baseia-se no monitoramento da cristalização de uma solução de PELBD. A concentração desta solução durante a cristalização numa varredura de temperatura é medida. Alíquotas da solução são filtradas e analisadas por um detector de concentração. O processo é similar ao fracionamento por precipitação; porém, neste caso, nenhuma atenção é dada ao polímero precipitado e sim ao polímero que continua na solução.

Quando a temperatura começa a diminuir, as frações do polímero que cristalizam mais rápido, compostas por moléculas com pouca ou nenhuma ramificação, irão precipitar primeiro, resultando num decréscimo na concentração da solução. Segue-se então a precipitação das frações de macromoléculas com maior quantidade de ramificações curtas, conforme a temperatura diminui. Os últimos pontos representam as frações que não cristalizaram e que continuam solúveis devido à elevada quantidade de ramificações curtas nas cadeias.

\section{Preparação dos filmes tubulares}

Os filmes foram processados numa extrusora de matriz anelar Carnevalli E-40 com abertura da matriz de $1,8 \mathrm{~mm}$. As principais condições de processamento destes filmes tubulares estão apresentadas na Tabela 2.

\section{Propriedades reológicas das resinas}

A temperatura utilizada em todos os ensaios foi a mesma utilizada na saída da matriz anelar durante o processamento dos filmes, $200{ }^{\circ} \mathrm{C}$.

Tabela 1. Descrição dos materiais utilizados no estudo.

\begin{tabular}{lcccccc}
\hline \multicolumn{1}{c}{ Polímero } & $\begin{array}{c}\text { I.F.* } \\
\mathbf{2 , 1 6} \mathbf{~ k g}\end{array}$ & $\begin{array}{c}\text { Densidade } \\
\mathbf{g} / \mathbf{c m}^{\mathbf{3}}\end{array}$ & $\begin{array}{c}\mathbf{M n} \\
\mathbf{g} / \mathbf{m o l}\end{array}$ & $\begin{array}{c}\mathbf{M w} \\
\mathbf{g} / \mathbf{m o l}\end{array}$ & $\begin{array}{c}\mathbf{M z} \\
\mathbf{g} / \mathbf{m o l}\end{array}$ & $\mathbf{M w / M n}$ \\
\hline PELBD-ZN1 & 1,18 & 0,9168 & 36.750 & 174.100 & 544.100 & 4,7 \\
PELBD-ZN2 & 1,06 & 0,9206 & 39.430 & 169.300 & 490.700 & 4,3 \\
m-PELBD & 0,99 & 0,9193 & 53.960 & 159.100 & 328.200 & 2,9 \\
\hline
\end{tabular}

*valores em g/10min medidos a $190{ }^{\circ} \mathrm{C}$. 
Tabela 2. Condições de processamento dos filmes tubulares de PELBD

\begin{tabular}{lccc}
\hline Espessura Filme & $60 \mu \mathrm{m}$ & $\begin{array}{c}\text { Altura Linha } \\
\text { Neve }\end{array}$ & $45 \mathrm{~cm}$ \\
\hline $\begin{array}{l}\text { Largura Filme/ } \\
\mathrm{R}_{\mathrm{f}} / \mathrm{R}_{\mathrm{o}}\end{array}$ & $51 \mathrm{~cm} / 2,2$ & $\begin{array}{c}\text { Temperatura Ar } \\
\text { Refrigerado }\end{array}$ & $17{ }^{\circ} \mathrm{C}$ \\
\hline $\begin{array}{l}\text { Rotação } \\
\text { Rosca/Motor }\end{array}$ & $1600 \mathrm{rpm}$ & $\begin{array}{c}\text { Pressão Ar } \\
\text { Refrigerado }\end{array}$ & $8 \mathrm{mmHg}$ \\
\hline $\begin{array}{l}\text { Velocidade } \\
\text { Puxamento }\end{array}$ & $13,5 \mathrm{~m} / \mathrm{min}$ & $\begin{array}{c}\text { Temperatura } \\
\text { Massa }\end{array}$ & $199-200^{\circ} \mathrm{C}$ \\
\hline $\begin{array}{l}\text { Pressão Rolo } \\
\text { Puxador }\end{array}$ & $2,5 \mathrm{bar}$ & Produtividade & $50,4 \mathrm{~kg} / \mathrm{h}$ \\
\hline
\end{tabular}

As medidas de $\eta(\dot{\gamma})$ a altas taxas, de $\dot{\gamma}_{c}$ e do inchamento do extrudado foram feitas num reômetro capilar Instron 4467 , com capilar de comprimento $6,0706 \mathrm{~cm}$ e diâmetro de $0,1519 \mathrm{~cm}$ segundo a norma ASTM D 3835. Para a verificação visual de $\dot{\gamma}$ foi utilizado um estereoscópio Motic, modelo SMZ-143 da DMI. Para a quantificação do inchamento do extrudado, os fundidos que saíam do capilar a cada taxa eram imersos em nitrogênio líquido. Os diâmetros dos extrudados foram medidos utilizando um micrômetro Mitutoyo modelo IP 54.

Medidas de $\mathrm{N}_{1}(\dot{\gamma})$ e $\eta(\dot{\gamma})$ a baixas $\dot{\gamma}$ foram feitas num reômetro ARES da Rheometrics,com placas paralelas de 25 mm de diâmetro e distância de $1 \mathrm{~mm}$ entre as placas.

As medidas de $\gamma_{r}(\tau, t), G^{\prime}(\omega)$ e G' $(\omega)$ foram obtidas usando um reômetro SR-200 da Rheometrics sob atmosfera de nitrogênio, com cone-placa de $25 \mathrm{~mm}$ de diâmetro, distância entre os dois de $0,048 \mathrm{~mm}$ e cone com ângulo de $0,01 \mathrm{rad}$.

\section{Morfologia e Rugosidade superficial}

A rugosidade superficial consiste em irregularidades na textura da superfície. Ela é uma quantificação do relevo topográfico da superfície. Um dos indicadores de rugosidade mais utilizado é a $R_{m s}{ }^{[15]}$ definida pela seguinte equação:

$$
\mathrm{R}_{\mathrm{ms}}=\sqrt{\frac{1}{\mathrm{~N}} \cdot \sum_{\mathrm{i}=1}^{\mathrm{N}} \mathrm{Z}_{\mathrm{i}}^{2}}
$$

onde $\mathrm{Z}_{\mathrm{i}}$ é a distância do i ponto até o "nível médio da superfície", o qual é definido por uma linha reta sobre a qual a rugosidade é medida. Esta linha é paralela à direção geral do perfil dentro dos limites do comprimento da amostra.

Para medir $\mathrm{R}_{\mathrm{ms}}$ e observar a textura dos filmes foi utilizado um microscópio de força atômica, MMA FM-2 da Digital Instruments, operando no modo contato. A medida da distribuição de tamanho dos domínios observados foi realizada com o software Image-pro Express 4.0.

A morfologia dos filmes foi analisada por microscopia eletrônica de varredura, MEV. Para aumentar o contraste entre a fase amorfa e cristalina foi feito um ataque químico nos filmes com solução de $\mathrm{KMnO}_{4}{ }^{[16]}$. A solução de ataque consistiu de uma mistura de água destilada com ácido o-fosfórico, ácido sulfúrico e $\mathrm{KMnO}_{4}$. A solução de contenção de ataque consistiu de uma mistura de água destilada com ácido sulfúrico e água oxigenada.

As amostras foram imersas na solução de ataque durante 5 min, secas em papel toalha e imersas em solução para con- ter o ataque durante $1 \mathrm{~min}$. Esse procedimento foi repetido quatro vezes para cada amostra. Guerrini ${ }^{[6]}$ verificou que o tempo ótimo de ataque para blendas de PELBD e PEBD era de $20 \mathrm{~min}$; logo esse tempo foi utilizado neste estudo. Após ataque as superfícies foram recobertas com ouro e analisadas em um MEV, modelo Philips XL-30 FEG.

\section{Propriedades Óticas dos filmes}

A opacidade total dos filmes tubulares foi medida de acordo com a ASTM D 1003. A opacidade é o espalhamento de luz em uma amostra, responsável pela redução no contraste de objetos vistos através dessa amostra, e corresponde ao percentual de luz transmitida que é espalhado em uma direção que desvia mais do que um ângulo especificado comparado à luz incidente. O ângulo especificado por essa norma é $2,5^{\circ}$ ou $0,044 \mathrm{rad}$.

A opacidade pode ser dividida em opacidade interna e externa. A interna é conseqüência do espalhamento de luz em regiões de diferentes índices de refração devido a diferentes arranjos morfológicos e graus de cristalinidade ${ }^{[16]}$. Já a opacidade externa ocorre devido a irregularidades da superfície. Essa rugosidade é afetada pelas propriedades reológicas do polímero, tipo de cristalização e superfície com a qual o fundido irá se solidificar. Sabe-se ainda que a opacidade externa é a que mais contribui para a opacidade total.

As análises de opacidade foram realizadas no equipamento Haze-gard plus da BYK Gardener de acordo com a norma ASTM D 1003 .

\section{Resultados e Discussão}

A Figura 2 mostra as curvas de DSCB para os três PELBD utilizados no estudo. As resinas $\mathrm{ZN}-1$ e ZN-2 apresentaram curvas típicas de PELBD catalisados com Ziegler-Natta ${ }^{[16,17]}$ com três regiões distintas: uma entre $\sim 72{ }^{\circ} \mathrm{C}$ e $88^{\circ} \mathrm{C}$, representando as populações de cadeias com pouca ou nenhuma ramificação curta, outra entre $30^{\circ} \mathrm{C}$ e $\sim 72{ }^{\circ} \mathrm{C}$ representando populações com uma quantidade média de ramificações curtas, e outra entre $25^{\circ} \mathrm{C} \mathrm{e} 30^{\circ} \mathrm{C}$, representando populações de moléculas que não cristalizam devido à grande quantidade de ramificações curtas. A Tabela 3 apresenta a percentagem de cada uma destas populações nos PELBD. Observa-se que

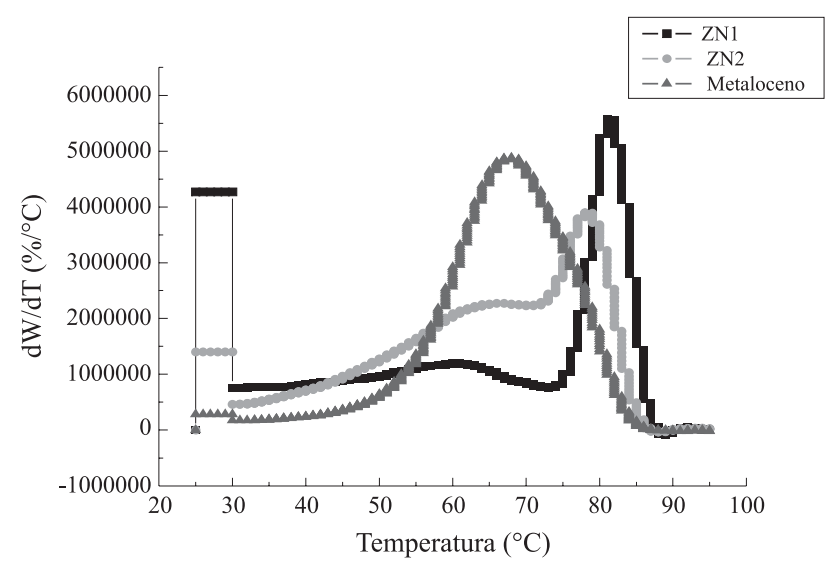

Figura 2. Distribuição de Ramificações curtas dos PELBD 
Tabela 3. Percentagem de cadeias com nenhuma ou pouca (A), com quantidade média (B) ou com muitas ramificações curtas (C) nos PELBD

\begin{tabular}{lccc}
\hline Polímero & $\begin{array}{c}\mathbf{A} \\
\text { (intervalo de temperatura) }\end{array}$ & $\begin{array}{c}\mathbf{B} \\
\text { (intervalo de temperatura) }\end{array}$ & $\begin{array}{c}\mathbf{C} \\
\text { (intervalo de temperatura) }\end{array}$ \\
\hline $\mathrm{ZN} 1$ & $35,7\left(75-88^{\circ} \mathrm{C}\right)$ & $41,3\left(30-75^{\circ} \mathrm{C}\right)$ & $23\left(25-30{ }^{\circ} \mathrm{C}\right)$ \\
$\mathrm{ZN} 2$ & $36,4\left(71,3-88^{\circ} \mathrm{C}\right)$ & $55,12\left(30-71,3{ }^{\circ} \mathrm{C}\right)$ & $8,5\left(25-30{ }^{\circ} \mathrm{C}\right)$ \\
$\mathrm{mPELBD}$ & $98,8\left(30-88^{\circ} \mathrm{C}\right)$ & $1.17\left(25-30{ }^{\circ} \mathrm{C}\right)$ \\
\hline
\end{tabular}

a resina $\mathrm{ZN}-1$ possui maior quantidade de cadeias altamente ramificadas do que a resina $\mathrm{ZN}-2$, que por sua vez, possui maior quantidade de cadeias moderadamente ramificadas do que a resina $\mathrm{ZN}-1$. A resina $\mathrm{mPELBD}$ apresentou uma DSCB Gaussiana (mais homogênea que as outras duas), com uma população grande de moléculas com nenhuma, pouca e média quantidade de ramificações curtas e uma população muito pequena de moléculas com muitas ramificações curtas .

A Figura 3a apresenta as curvas de $\eta(\dot{\gamma})$ entre $0.01 \mathrm{e}^{2} 00 \mathrm{~s}^{-1}$ para os PELBD. Pode-se verificar que não existe diferença entre o comportamento das resinas $\mathrm{ZN}-1$ e $\mathrm{ZN}-2$ apesar de possuírem diferentes DSCB. Com relação ao $\mathrm{mPELBD}$, observa-se menor pseudo plasticidade $(n \rightarrow 1)$ do que nas outras duas resinas. Esta diferença, porém, não pode ser atribuída somente a sua diferente DSCB já que esta resina também possui uma DPM mais estreita que os outros PELBD e sabese que essa região da curva de viscosidade é mais sensível a variações de $\mathrm{DPM}^{[5]}$; quanto mais estreita a DPM menos pseudoplástico é o polímero fundido.
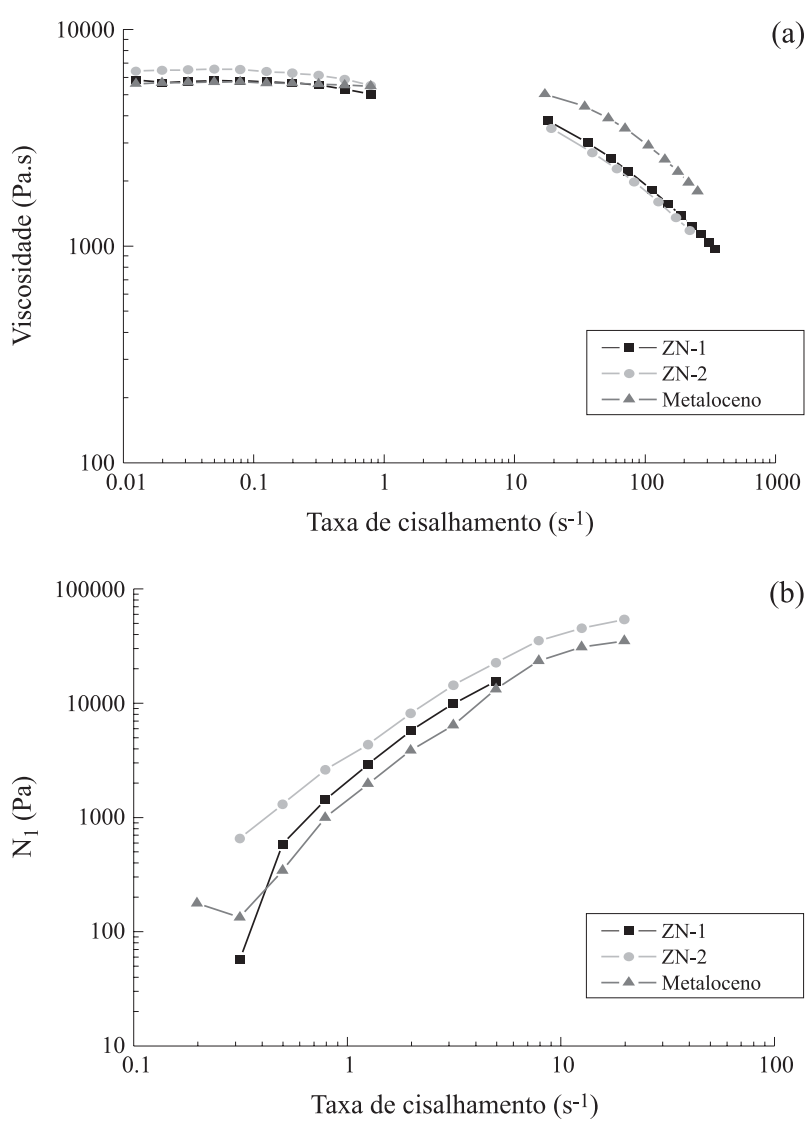

Figura 3. a) Viscosidade $x$ Taxa de cisalhamento dos PELBD, b) $N_{1}$ x Taxa de cisalhamento dos PELBD.

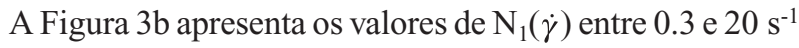
para os PELBD. Observa-se que a resina $\mathrm{ZN}-2$ apresenta maiores valores de $\mathrm{N}_{1}$ do que a resina $\mathrm{ZN}-1$. Um maior valor de $\mathrm{N}_{1}$ significa que a resina possui maior elasticidade. Em relação à resina $\mathrm{ZN}-1$, a resina $\mathrm{ZN}-2$ possui similar quantidade de moléculas altamente lineares, mas maior percentagem de cadeias com quantidade média de ramificações e bem menor quantidade de cadeias altamente ramificadas. Assim, a sua maior elasticidade pode ser atribuída a esta fração maior de cadeias com número médio de ramificações.

A Figura 4 apresenta os valores de inchamento do extrudado em função da taxa de cisalhamento entre 3 e $140 \mathrm{~s}^{-1}$ para os PELBD. Observa-se que até $60 \mathrm{~s}^{-1}$, o inchamento do extrudado aumenta com a taxa de cisalhamento e que as resinas ZN's apresentam inchamentos superiores aos da resina metalocênica, comportamentos estes similares ao comportamento da $\mathrm{N}_{1}(\dot{\gamma})$. Porém, a taxas acima de $60 \mathrm{~s}^{-1}$, a resina $\mathrm{ZN}-1$ apresenta inchamento superior ao da resina $\mathrm{ZN}-2$, já que possui maior quantidade de moléculas com quantidade elevada de ramificações curtas. O máximo de inchamento da resina $\mathrm{ZN}-2$ é obtido a $60 \mathrm{~s}^{-1}$; após esta taxa, o inchamento diminui, como esperado ${ }^{[5]}$.

Os valores de $\dot{\gamma}$ estão apresentados na Tabela 4 enquanto que a Figura 5 mostra a superfície dos extrudados durante a fratura. Observa-se que o mPELBD fratura à taxas menores do que as resinas $\mathrm{ZN}-1$ e $\mathrm{ZN}-2$, provavelmente devido à sua DPM mais estreita. Nas resinas de DPM mais larga existe maior quantidade de moléculas de baixo peso molecular, as quais atuam como facilitadoras de fluxo das moléculas de maior PM ajudando no deslizamento de umas sobre as outras,

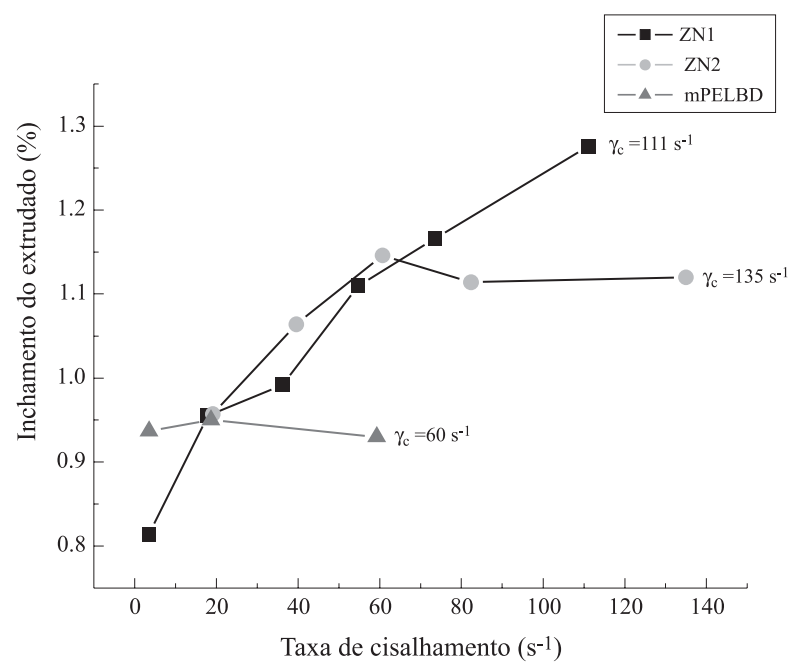

Figura 4. Inchamento do extrudado x Taxa de cisalhamento, mostrando $\dot{\gamma}_{c}$ dos PELBD. 

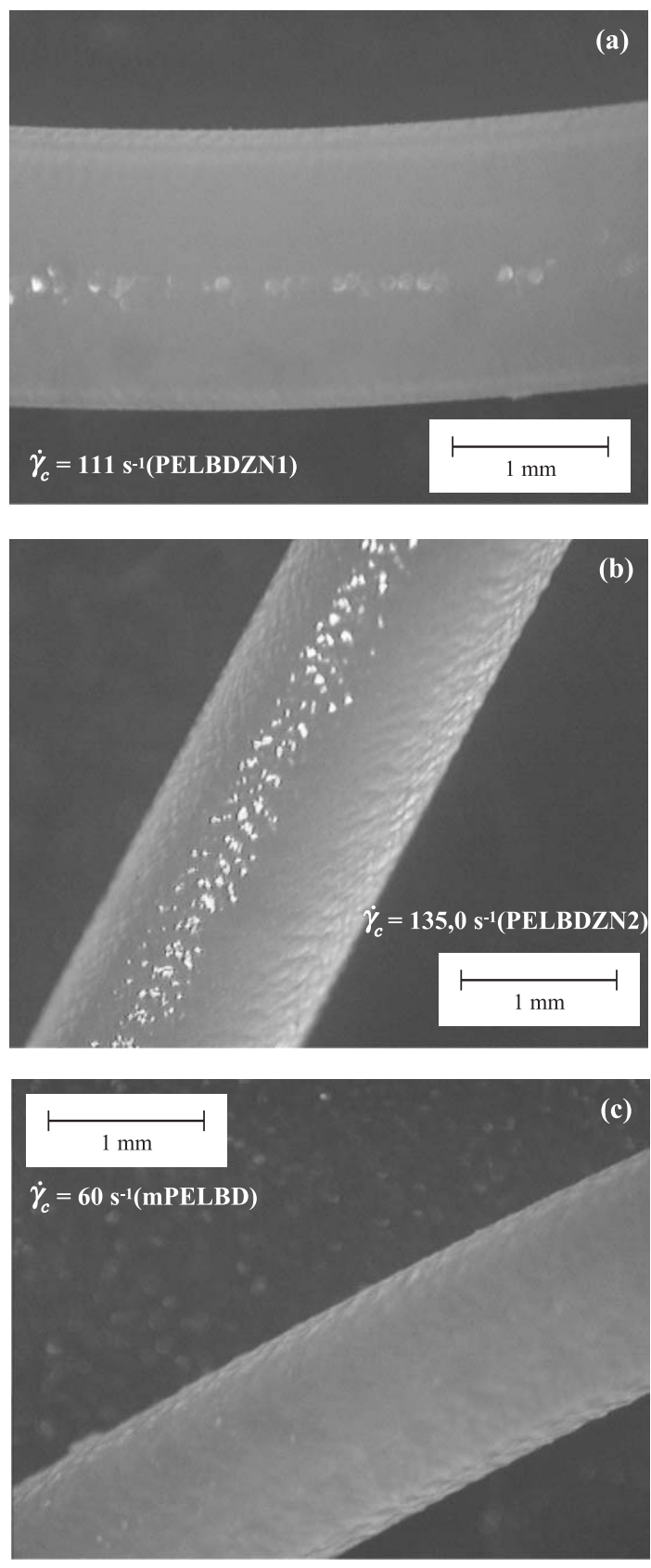

Figura 5. Fratura do fundido dos PELBD.

diminuindo o tempo para relaxação das mesmas e a tensão sobre a amostra. Quanto menor o tempo de relaxação, mais rapidamente as deformações sofridas no capilar são recuperadas, diminuindo assim os efeitos de superfície e adiando o aparecimento da fratura do fundido para $\dot{\gamma}$ mais elevadas. Já a fratura a taxas de cisalhamento menores da $\mathrm{ZN}-1$, em relação à $\mathrm{ZN}-2$, pode ser creditada à sua maior quantidade de moléculas com muitas ramificações curtas, as quais aumentam o tempo de relaxação da resina, diminuindo então $\dot{\gamma}$.

A Figura 6a apresenta as curvas de $G^{\prime}(\omega)$, entre 0.01 e 300 $\mathrm{rad} / \mathrm{s}$, para os PELBD. Pode-se observar que a baixas freqüências, o $\mathrm{ZN}-2$ possui valores de G' levemente superiores aos dos outros polietilenos, possivelmente devido à sua maior quantidade de frações de moléculas com quantidade média de ra-
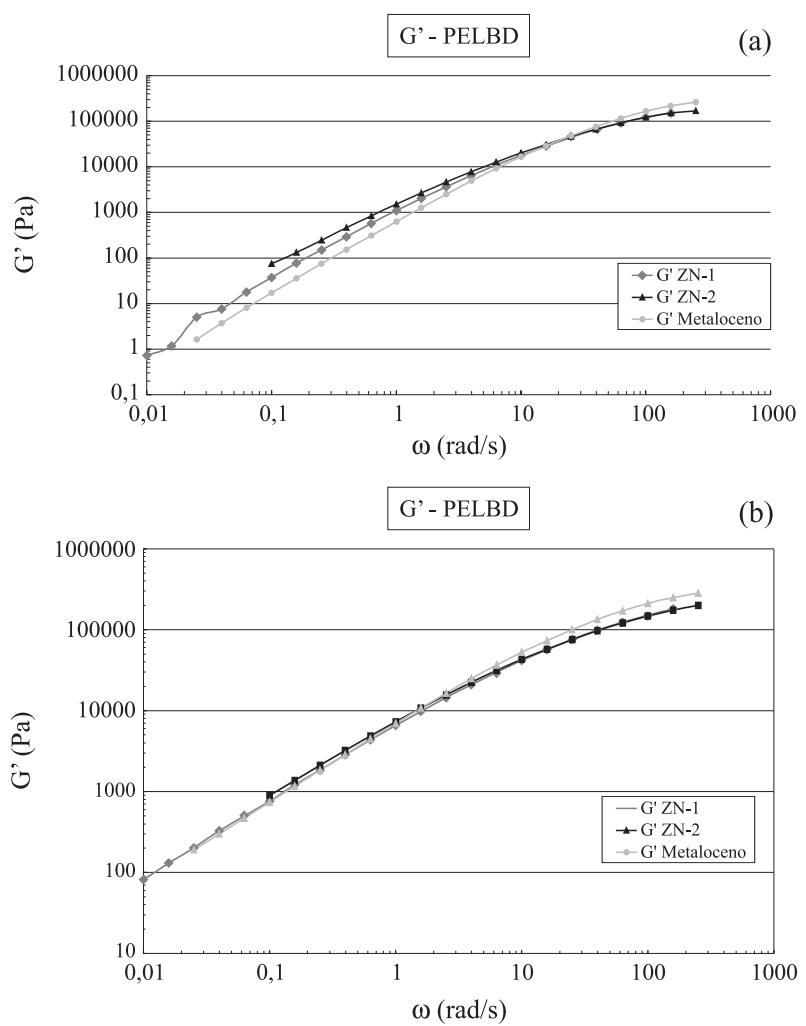

Figura 6. a) Valores de G'(w) dos PELBD; b) Valores de G'(w) dos PELBD.

mificações curtas. Quanto maior G', maior será a estabilidade da bolha durante o processamento dos filmes tubulares ${ }^{[6,18]}$; assim, espera-se que a bolha formada pelo PELBD ZN-2 seja mais estável do que a formada pelo PELBD ZN-1. O metaloceno foi o que apresentou menores valores de G' a baixas freqüências. Neste caso, esse desempenho não pode ser creditado somente ao fato da sua DSCB ser mais homogênea já que essa resina possui também uma DPM mais estreita que os PELBD-ZN o que pode ter ocasionado essa diferença no comportamento de G'. Fang et al. ${ }^{[18]}$ encontraram comportamentos similares para PELBD-ZN e m-PELBD com relação ao G', porém parâmetros como PM, DPM e DSCB dos materiais estudados nessa comparação não foram medidos.

A Figura 6b mostra as curvas de G" $(\omega)$ para os PELBD. Pode-se observar que os PELBD-ZN possuem valores de G" similares, ou seja, a DSCB não teve influência nessa propriedade, como também verificado nas medidas de $\eta(\dot{\gamma})$. Esse comportamento era esperado uma vez que o PM e a DPM dos polímeros estudados são similares. O m-PELBD apresentou valores de G" ligeiramente maiores a partir de $10 \mathrm{rad} / \mathrm{s}$, da mesma forma que a sua $\eta(\dot{\gamma})$ teve valores maiores a altas taxas de cisalhamento, devido à sua DPM ser mais estreita. Logo, este comportamento de G" da resina metalocênica deve ser creditado às diferenças em DPM entre ela e as resinas ZN.

A Figura 7 mostra as curvas de fluência durante a aplicação de uma tensão de $500 \mathrm{~Pa}$ por 300 s e recuperação da deformação quando essa tensão é retirada. A Tabela 4 também mostra os valores de $\gamma_{\mathrm{r}}$ calculados pela seguinte expressão:

$$
\gamma_{\mathrm{r}}=\frac{\gamma(300 \mathrm{~s})-\gamma(900 \mathrm{~s})}{\gamma(300 \mathrm{~s})}
$$




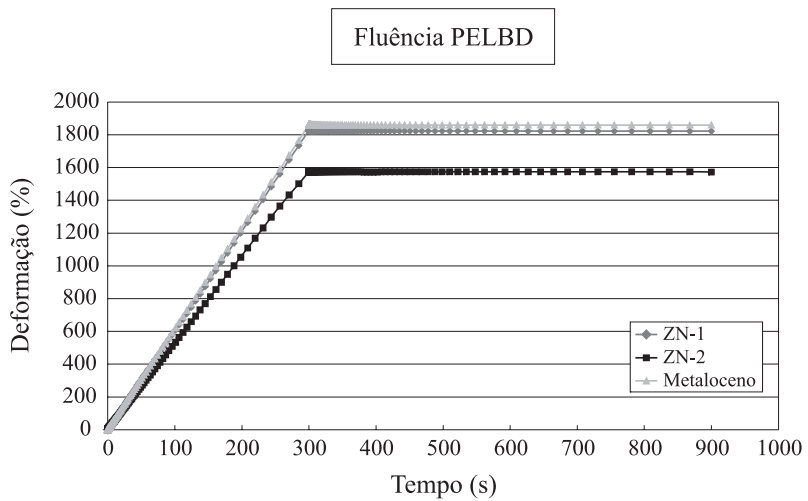

Figura 7. Fluência e deformação recuperável dos PELBD.
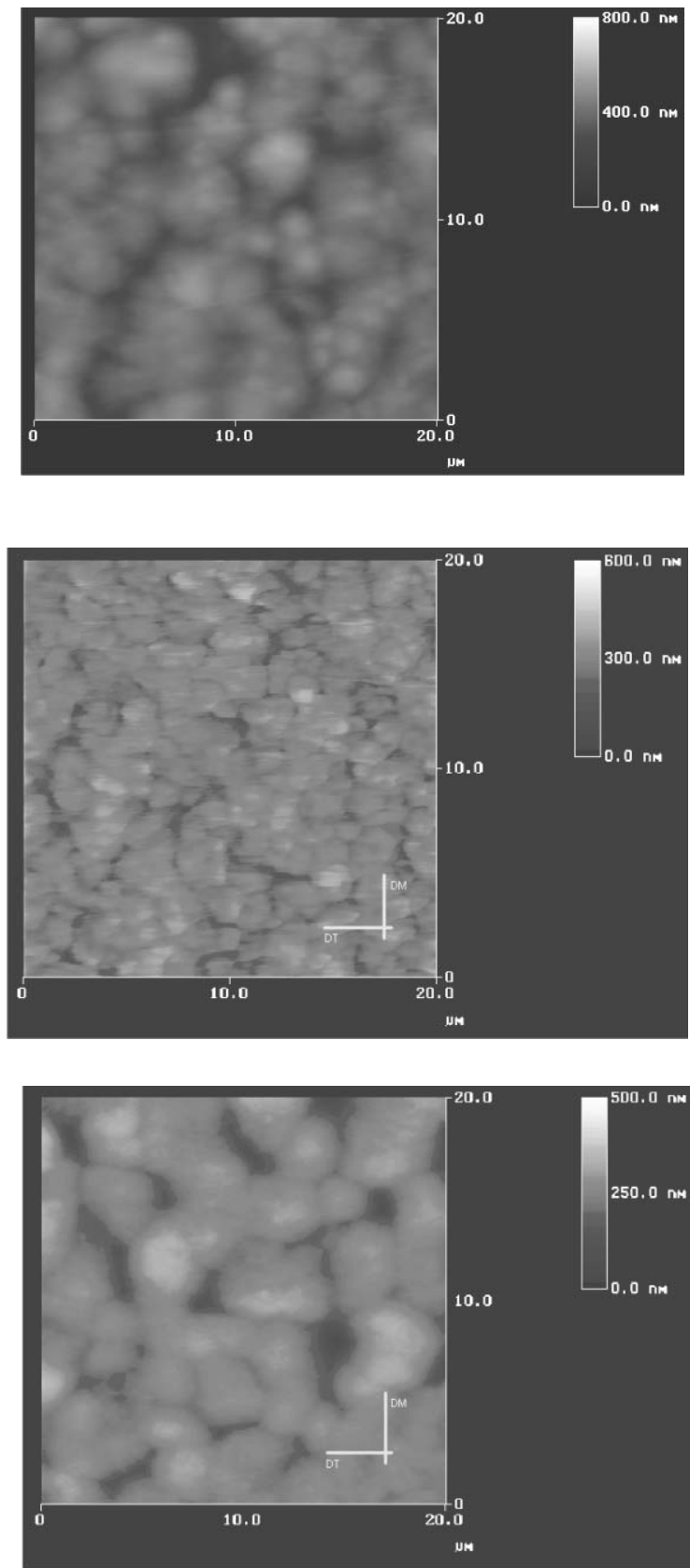

(b)

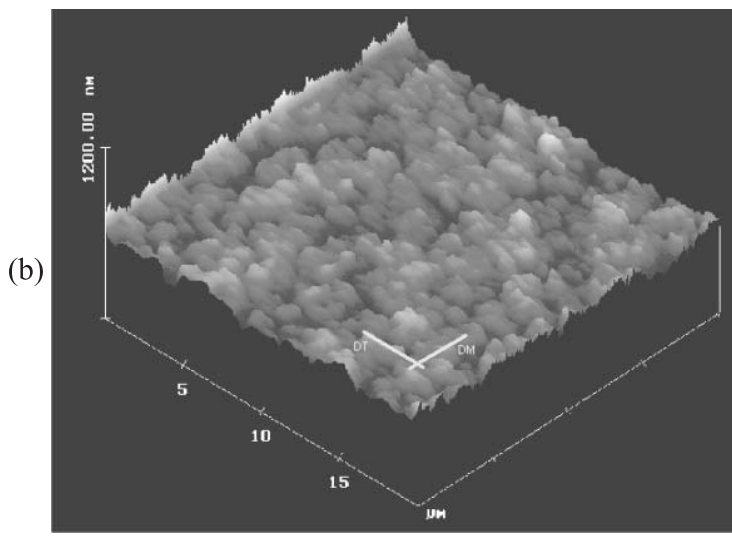

(c)

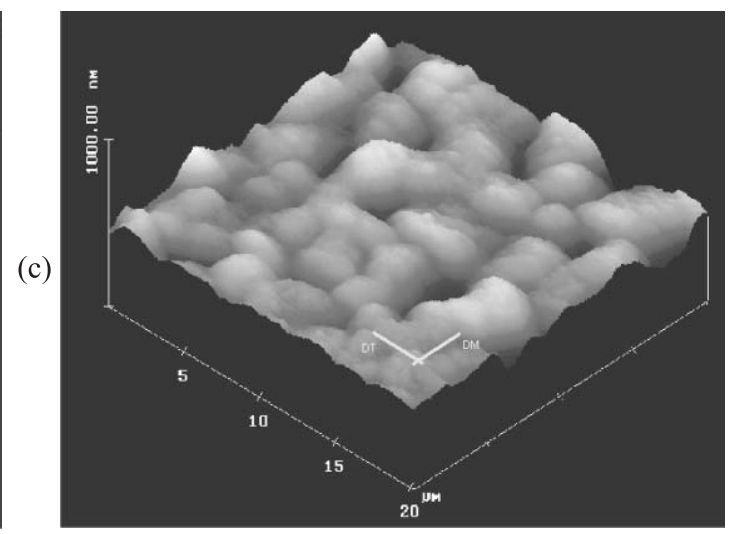

Figura 8. a) Superfície e topografia do filme de PELBDZN-1; b) Superfície e topografia do filme de PELBDZN-2; c) Superfície e topografia do filme de mPELBD.

onde (300s) é a deformação após 300s (sob tensão constante de 500Pa) e $\gamma(900$ s) é a deformação após 900s (após retirada da tensão em $300 \mathrm{~s}$ ).

A resina $\mathrm{ZN}-1$ se deforma mais do que a $\mathrm{ZN}-2$, o que era de se esperar porque possui maior quantidade de cadeias altamente ramificadas (facilitadoras do fluxo). O mPELBD também apresenta uma deformação maior do que o $\mathrm{ZN}-2$; além da DSCB, esta resina possui PM e DPM diferentes dos das resinas ZN. Esta elevada deformação pode ser atribuída aos seus menores $\mathrm{M}_{\mathrm{w}}$ e $\mathrm{M}_{\mathrm{z}}$.

Em relação à recuperação dessa deformação, observa-se ainda que o mPELBD apresenta menor recuperação da deformação, ou seja, recupera lentamente a deformação; assim,

(a)

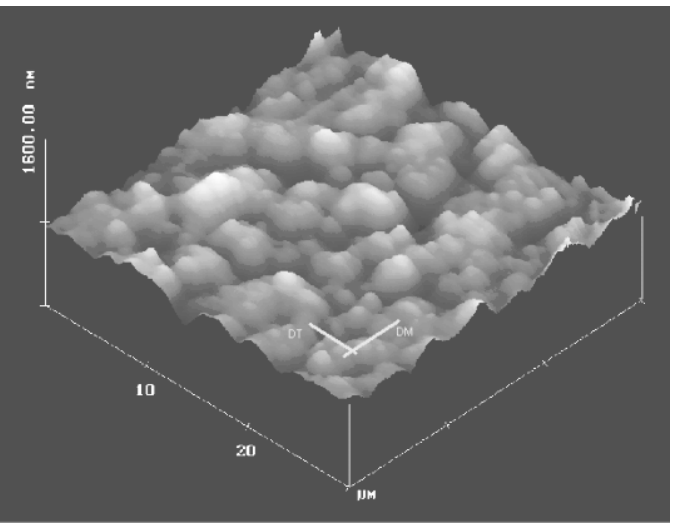


espera-se que devido a esta lenta recuperação, a sua cristalização ocorra sob a influencia das tensões biaxiais o que levaria à formação de cilindritos, com lamelas empilhadas espessas. Por outro lado a ZN-1 apresentou a maior deformação
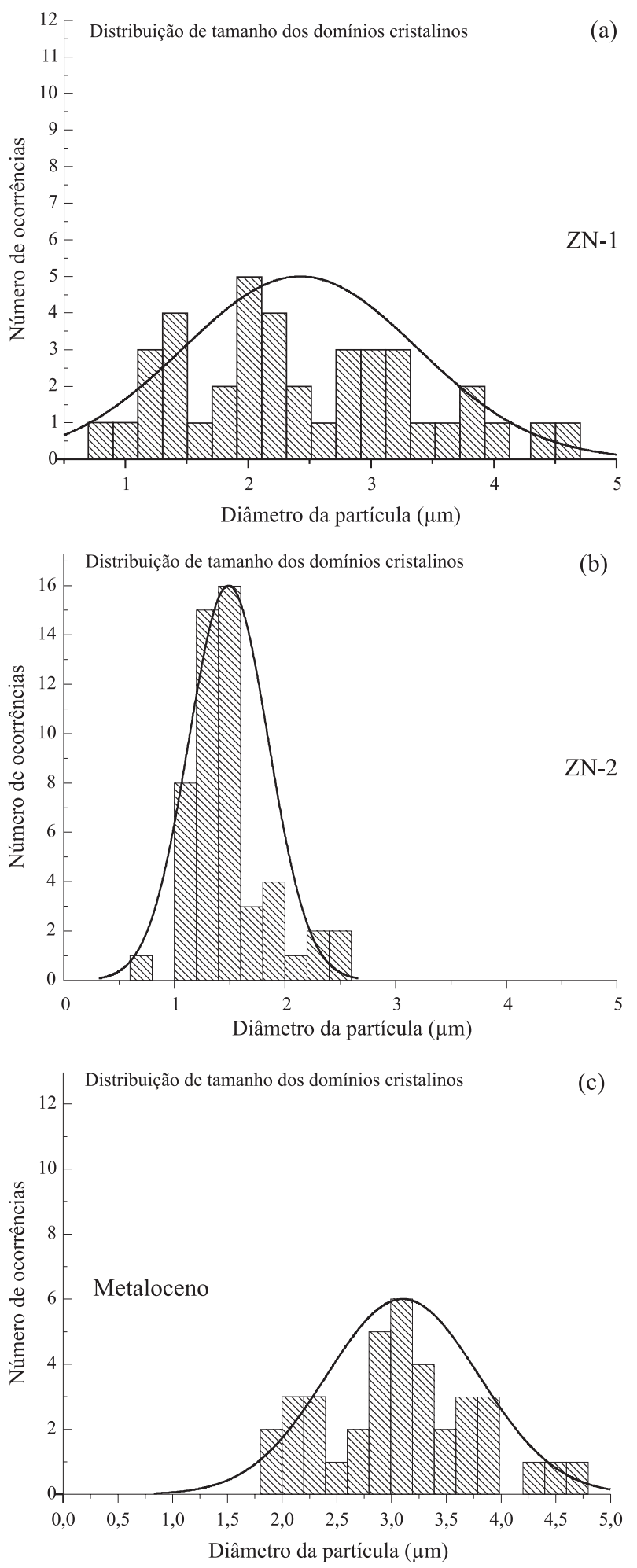

Figura 9. a) Distribuição de tamanho dos domínios da superfície do filme de PELBDZN-1; b) Distribuição de tamanho dos domínios da superfície do filme de PELBDZN-2; c) Distribuição de tamanho dos domínios da superfície do filme de mPELBD. recuperável; espera-se então que este tempo menor para a recuperação faça com que a sua cristalização ocorra sem a influencia das tensões biaxiais o que produziria cristalitos pequenos. Assim esperar-se-ia que devido à $\gamma_{\mathrm{r}}$, a opacidade do $\mathrm{mPELBD}>\mathrm{ZN}-2>\mathrm{ZN}-1$. Esta proporcionalidade se manteria, porém, se efeitos reológicos como a fratura do fundido e pele de cação não aparecessem antes durante a extrusão e se sobrepusessem aos efeitos da cristalização sob tensão. A $\mathrm{ZN}-1$ apresentou fratura do fundido a uma taxa menor do que a ZN2; logo, nesse caso, a fratura do fundido se sobrepôs
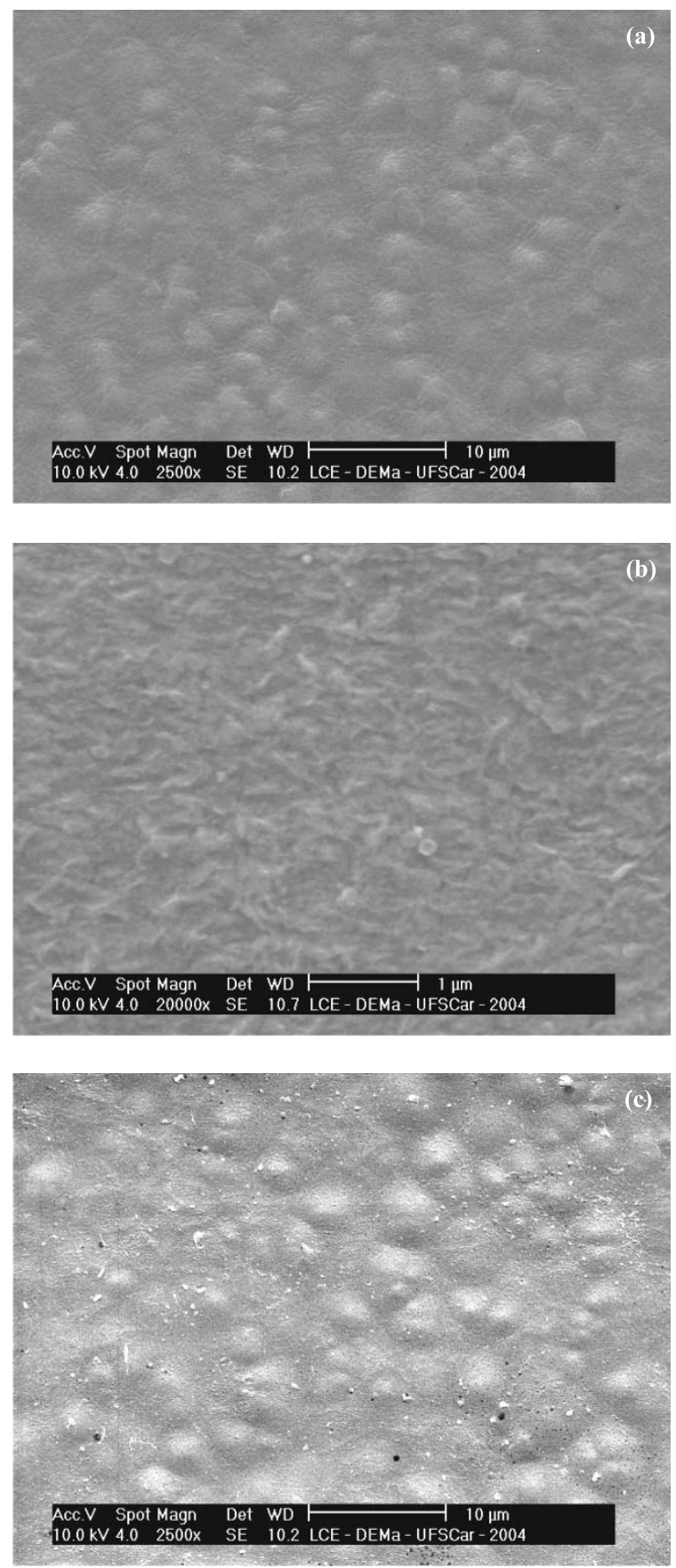

Figura 10. a) Micrografia de MEV do filme de PELBDZN-1(2500x). b) Micrografia de MEV do filme de PELBDZN-2(20000x). c) Micrografia de MEV do filme de mPELBD(2500x). 
Tabela 4. Valores de e $\gamma_{\mathrm{r}}$ dos PELBD e da $\mathrm{R}_{\mathrm{ms}}$ e opacidade dos filmes soprados de PELBD

\begin{tabular}{lcccc}
\hline Materiais & $\dot{\gamma}_{\mathbf{c}}$ & $\boldsymbol{\gamma}_{\mathrm{r}}$ & $\mathbf{R}_{\mathrm{ms}}$ dos filmes & $\begin{array}{c}\text { Opacidade total } \\
\text { dos filmes } \\
(\mathbf{\%})\end{array}$ \\
\hline Metaloceno & 60 & 0,105 & 39,442 & 19,5 \\
$\mathrm{ZN}-2$ & 135 & 0,189 & 36,626 & 11,2 \\
$\mathrm{ZN}-1$ & 111 & 0,212 & 49,752 & 25,2 \\
\hline
\end{tabular}

à recuperação da deformação e a opacidade terá o seguinte comportamento: $\mathrm{mPELBD}>\mathrm{ZN}-1>\mathrm{ZN}-2$.

A Figura 8 apresenta micrografias de AFM da superfície dos filmes de PELBD, enquanto a Tabela 4 apresenta os valores de rugosidade. Observa-se que os domínios do $\mathrm{ZN}-1$ e do mPELBD são maiores do que os domínios do $\mathrm{ZN}-2$ e que a superfície mais rugosa é a do $\mathrm{ZN}-1$. A distribuição de tamanho destes domínios é mostrada na Figura 9. O ZN-1 possui uma distribuição do tamanho de domínios entre $0,5 \mathrm{e}$ $4,7 \mu \mathrm{m}$, a ZN-2 entre 0,6 e 2,6 $\mu \mathrm{m}$ e o Metaloceno entre 1,8 e 4,5 $\mu \mathrm{m}$; logo a distribuição de tamanhos dos domínios da resina $\mathrm{ZN}-2$ é mais estreita do que a das outras duas.

A Figura 10 apresenta as micrografias de MEV destes PELBD. Pode-se observar novamente que as estruturas do ZN-1 e do mPELBD são globulares, sem orientação aparente e bem maiores do que as estruturas do $\mathrm{ZN}-2$.

Resumindo, então, conclui-se que os domínios dos filmes das resinas ZN-1 e mPELBD são maiores e mais rugosos do que os domínios dos filmes da resina $\mathrm{ZN}-2$, possuindo ainda uma distribuição de tamanhos mais larga. A partir destes resultados, espera-se então que, pela analise da superfície dos filmes, a opacidade do $\mathrm{mPELBD} \approx \mathrm{ZN}-1>\mathrm{ZN}-2$.

A Tabela 4 também apresenta os valores de opacidade total dos filmes de PELBD. Os filmes de ZN-1 e mPELBD apresentam maior opacidade total que os filmes de $\mathrm{ZN}-2$. A Figura 11 mostra a correlação entre a opacidade total e $\gamma_{\mathrm{r}}$

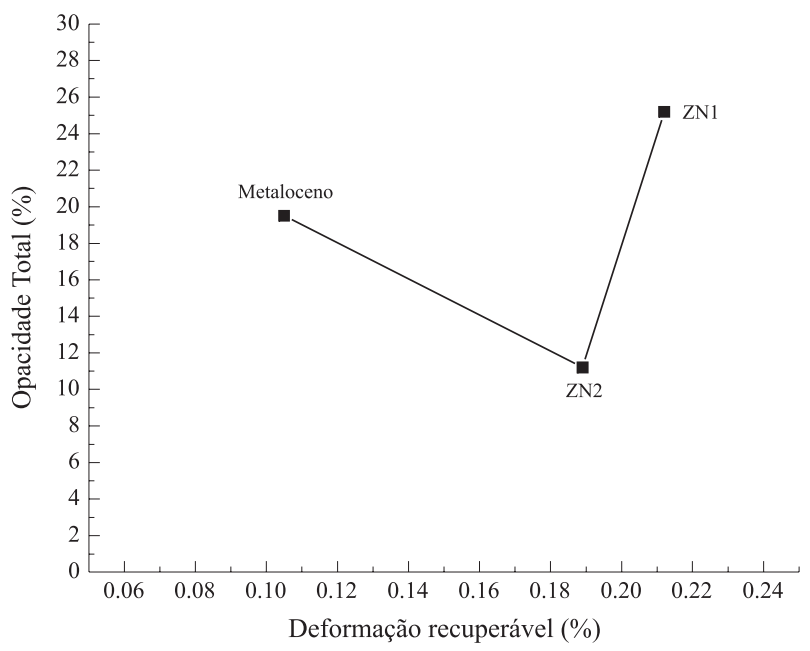

Figura 11. Correlação entre a opacidade total e a deformação recuperável para os PELBD. para estes PELBD. Assim, embora a resina ZN-1 tenha apresentado valores maiores de $\gamma_{\mathrm{r}}$ do que a resina $\mathrm{ZN}-2$, a sua opacidade total foi maior porque o extrudado fraturou antes.

\section{Conclusões}

A partir destes resultados pode-se concluir:

a. Para resinas com similares PM e DPM, a DSCB exerce influencia nas propriedades reológicas que refletem a elasticidade do material, como $\mathrm{N}_{1}(\dot{\gamma}), \mathrm{G}^{\prime}(\omega)$ e $\gamma_{\mathrm{r}}$, porém não exerce influencia em propriedades reológicas que refletem a viscosidade do material como $\eta(\dot{\gamma})$ e G' $(\omega)$.

b. Para uma dada resina, a DPM influenciará mais nos valores de $\eta(\dot{\gamma})$ do que a sua DSCB.

c. Para predizer as propriedades óticas de uma resina a partir das propriedades reológicas, torna-se necessário medir tanto a deformação recuperável como a fratura do fundido, para observar se esta última se sobrepõe à deformação recuperável.

\section{Referências Bibliográficas}

1. Tso, C. C., Deslauriers, P.J.-Polymer, 45, p. 2657(2004).

2. Gabriel, C., Lilge, D.-Polymer, 42, p. 297 (2001).

3. Coutinho, F., Mello, I., Santa Maria, L.-Polímeros: Ciência e Tecnologia, 13, $\mathrm{n}^{\circ}$ 1, p. 1(2003).

4. Middleman, S.-'Fundamentals of Polymer Processing", McGrawHill, New York, (1977).

5. Bretas, R.E.S. e Dávila, M.A.. -"Reologia de Polímeros Fundidos", 2a ed., EDUFSCar, São Carlos, SP (2005)

6. Guerrini, L.M.,-"Correlação entre propriedades reológicas e propriedades óticas de filmes de polietileno soprados", Dissertação de mestrado, Universidade Federal de São Carlos, Brasil (2003).

7. Taylor, J., Baik, J.J.-J. Plastic Film \& Sheeting, 16, p. 223 (2000).

8. Sukhadia, A. M., Rholfing, D., Johnson, M. Wilkes, G.J.Appl.Polym.Sci.,85, n. 11, p. 2396 (2002).

9. Sukhadia, A. M.-J.Plastic Film \& Sheeting,16, p. 54(2000).

10. Kazatchkov, I., Bohnet, N., Goyal, S.K., Hatzikiriakos, S.G.Polym.Eng.Sci., 39, n. 4, p.804 (1999).

11. Lu, J.,-J.Rheol., 42, p. 408 (1998).

12. Mackley, M. R.-J.Non-Newt.Fluid Mech.,76, p. 281(1998).

13. Santamaría, A., Fernández, M., Sanz, E, Lafuente, P., MuñozEscalona, A.-Polym., 44, p. 2473(2003).

14. Britto, L. Soares, J. Penlidis, A., Monrabal, B.-J.Polym.Sci.: Part B: Polym. Phys.,37, p. 539 (1999).

15. Kamal, M.R., Tang, Z., Huang, T.-International Polym. Process., XVI, p. 376 (2001).

16. Peacook, A. J., Handbook of polyethylene: structure, properties and applications, Marcel Dekker Inc., New York (2000).

17. Monrabal, B.-New Trends in Polyolefin Science and Technology, p. 119 (1996).

18. Fang, Y.,Carreau, P.J., Lafleur, P.G.-"Rheological effects in film blowing", in: Anais - Annual Meeting of the Polymer Processing Society, PPS-17, Montreal (2001).

Enviado: 07/10/05

Reenviado: 18/01/06

Aprovado: 27/01/06 